MS-TP-01-7, IFUP-TH 2001/31

\title{
Hamiltonian Monte Carlo simulation of the two-dimensional Wess-Zumino model
}

\author{
Matteo Beccaria ${ }^{\mathrm{a}}$, Massimo Campostrini ${ }^{\mathrm{b} *}$ and Alessandra $\mathrm{Feo}^{\mathrm{c}}$ \\ âDipartimento di Fisica dell'Università di Lecce and I.N.F.N., Sezione di Lecce \\ ${ }^{\text {b}}$ Dipartimento di Fisica dell'Università di Pisa and I.N.F.N., Sezione di Pisa \\ ${ }^{\mathrm{c} I n s t i t u t ~ f u ̈ r ~ T h e o r e t i s c h e ~ P h y s i k, ~ W e s t f a ̈ l i s c h e ~ W i l h e l m s-U n i v e r s i t a ̈ t, ~ M u ̈ n s t e r ~}$
}

We study a Hamiltonian lattice version of the two-dimensional Wess-Zumino model. Preliminary results obtained by Quantum Monte Carlo with a many-parameter guiding wave function are presented. We analyze the pattern of supersymmetry breaking by measuring the ground state energy and a set of supersymmetric Ward identities. The algorithm is quite effective and allows very precise measurements.

\section{INTRODUCTION}

Numerical simulations of lattice field theories are usually performed in the Lagrangian formulation. The alternative approach of the Hamiltonian formulation [1] has received very little attention so far. Nonetheless, we think there are very good reasons to develop numerical simulation techniques for the Hamiltonian approach: powerful many-body techniques are available [2], which allow the direct computation of the vacuum wave function properties; fermions are implemented directly and need not be integrated out; property like the mass spectrum are more immediate. A check of universality between the Lagrangian and the Hamiltonian formalism would also be very welcome.

\section{THE MODEL}

The continuum two-dimensional Wess-Zumino model is defined by the supersymmetry charges

$$
\begin{aligned}
Q_{1,2}=\int \mathrm{d} x\left[p(x) \psi^{1,2}(x)\right. & \\
& \left.-\left(\frac{\partial \phi}{\partial x} \pm V(\phi(x))\right) \psi^{2,1}(x)\right],
\end{aligned}
$$

where $\phi(x)$ is a real scalar field, $p(x)$ is its conjugate momentum, and $\psi(x)$ is a Majorana fermion.

*Presented by M. Campostrini
The charges obey the $N=1$ supersymmetry algebra

$Q_{1}^{2}=Q_{2}^{2}=P^{0} \equiv H, \quad\left\{Q_{1}, Q_{2}\right\}=2 P^{1} \equiv 2 P$,

which we have written in the Majorana basis $\gamma_{0}=C=-\sigma_{2}, \gamma_{1}=\mathrm{i} \sigma_{3}$. Since $P$ is not conserved on the lattice, a lattice formulation of a supersymmetric model must break the algebra explicitly. A very important advantage of the Hamiltonian formulation is that, since $H$ is conserved exactly, we can maintain a 1-dimensional supersymmetry subalgebra, e.g., $Q_{1}^{2}=H$, and expect to recover the rest in the continuum limit, together with Lorentz invariance [3]; this subalgebra is enough to guarantee some of the most important property of supersymmetry, including pairing of fermionic and bosonic states of nonzero energy; spontaneous breaking of supersymmetry is equivalent to nonzero ground-state energy $E_{0}$.

We adopt the lattice formulation [4]

$$
\begin{aligned}
Q \equiv Q_{1}=\sum_{n=1}^{L}[ & p_{n} \psi_{n}^{1} \\
& \left.-\left(\frac{\phi_{n+1}-\phi_{n-1}}{2}+V\left(\phi_{n}\right)\right) \psi_{n}^{2}\right],
\end{aligned}
$$

with canonical (anti)commutation rules, and $H=$ $Q^{2}$. We can replace the Majorana fields $\psi^{1,2}$ by one Dirac field $\chi$ by the clever transformation [4] $\psi_{n}^{1,2}=\frac{(-1)^{n} \mp \mathrm{i}}{2 \mathrm{i}^{n}}\left(\chi_{n}^{\dagger} \pm \mathrm{i} \chi_{n}\right)$, 
obtaining the Hamiltonian

$$
\begin{aligned}
& H=\frac{1}{2} \sum_{n=1}^{L}\left[p_{n}^{2}+\left(\frac{\phi_{n+1}-\phi_{n-1}}{2}+V\left(\phi_{n}\right)\right)^{2}\right. \\
& \left.-\left(\chi_{n}^{\dagger} \chi_{n+1}+\text { h.c. }\right)+(-1)^{n} V^{\prime}\left(\phi_{n}\right)\left(2 \chi_{n}^{\dagger} \chi_{n}-1\right)\right]
\end{aligned}
$$

which is explicitly free of sign problems, except potentially at the boundary; we adopt free boundary conditions to avoid the sign problem altogether [5].

In strong coupling, the model reduces to a supersymmetric quantum mechanics for each site; supersymmetry is broken if and only if the degree of the prepotential $V$ is even $\| 6$. In the continuum (and on the lattice in weak coupling), supersymmetry is broken at tree level if and only if $V$ has no zeroes. The predictions of strong coupling and weak coupling are quite different, and it is interesting to study both numerically and analytically the crossover from strong to weak coupling.

As a benchmark of the case where supersymmetry is unbroken, we consider the cubic prepotential $V=\phi^{3}$. In the more interesting case of broken supersymmetry we study the quadratic prepotential $V=\phi^{2}+\lambda_{0}$. Here, spontaneous symmetry breaking is expected to occur at $\lambda_{0}>0$, while for $\lambda_{0}<0$ the scenario is less clear; in fact, weak coupling perturbation theory predicts a symmetric ground state while strong coupling predicts broken supersymmetry with $E_{0} \sim \exp \left(c \lambda_{0}\right)$ for $\lambda_{0} \rightarrow-\infty$.

\section{MONTE CARLO SIMULATIONS}

We perform our simulations by the Green Function Monte Carlo algorithm [2] with the guiding wave function associated to the trial ground state

$$
\begin{aligned}
\left|\Psi_{0}\right\rangle_{\text {trial }} & =\exp \left[\sum_{n} F_{n}\right]\left|\Psi_{0}\right\rangle_{\text {free }}, \\
F_{n} & =P_{1}\left(\phi_{n}\right)+(-1)^{n}\left(\chi_{n}^{\dagger} \chi_{n}-1 / 2\right) P_{2}\left(\phi_{n}\right),
\end{aligned}
$$

where $\left|\Psi_{0}\right\rangle_{\text {free }}$ is the free $(V=0)$ ground state and $P_{1,2}$ are polynomials optimized according to the algorithm described in Ref. [7]; see Ref. [5] for more details. We wish to remark that, in order to keep the variance of observables finite as the simulation proceeds, it is necessary to simulate a

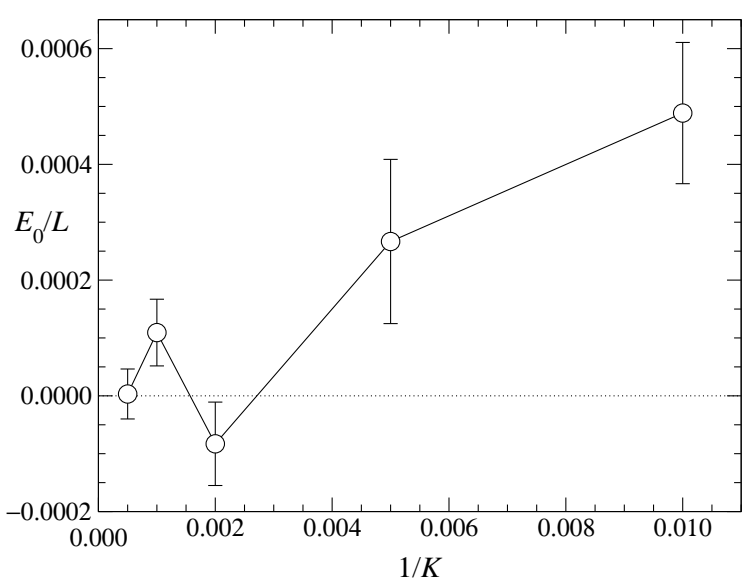

Figure 1. $E_{0} / L$ vs. $1 / K$ for $V=\phi^{3}$.

population of $K$ walkers (field configurations at fixed time), and extrapolate the results to $K \rightarrow$ $\infty$.

The trial wave function is an important feature of our simulations, and we are able to determine accurately the free parameters. The trial wave function allows a substantially improvement of the quality of our numerical results; moreover, since it is an approximation to the exact ground state, it contains important physical information about the model.

This algorithm can be parallelized effectively on a network of PCs connected through Ethernet; our MPI code reaches $90 \%$ efficiency.

We measure the ground-state energy $E_{0}$, which is the simplest and most precise observable to measure in a Quantum Monte Carlo, and a number of observables of the form $\left\{Q, X_{q}\right\}, X_{q}=$ $\sum_{n} \phi_{n}^{q} \psi_{n}^{2}$, which are zero for unbroken supersymmetry (for any fermionic operator $X,\langle\{Q, X\}\rangle=$ 0 is a supersymmetry Ward identity).

Fig. 11 shows the ground-state energy per site as a function of $1 / K$ for the cubic prepotential $V=\phi^{3}$ and $L=10$. The evidence for unbroken supersymmetry is quite convincing. The bosonic and fermionic contribution to $E_{0} / L$ are $\simeq \pm 0.7$ : we are observing a cancellation of the order of $10^{-4}$. Similar conclusions can be drawn by looking at $\left\{Q, X_{q}\right\}$, with smaller numerical accuracy. 
Fig. 2 shows the ground-state energy per site as a function of $1 / K$ for the quadratic prepotential $V=\phi^{2}+\lambda_{0}$, for $\lambda_{0}=-1,-1.25,-1.5$, and -2 and $L=10,22$. The data are insensitive to the value of $L$, and they are consistent with a linear extrapolation to $1 / K \rightarrow 0$. Supersymmetry breaking is clear for $\lambda_{0} \geq-1.25$; for smaller values of $\lambda_{0}$, we cannot discriminate between $E_{0}=0$ and an exponentially decreasing $E_{0}$. As in the cubic case, the study of $\left\{Q, X_{q}\right\}$ gives similar conclusions, with smaller numerical accuracy.

\section{CONCLUSIONS}

The Hamiltonian approach is a worthwhile alternative to the usual Lagrangian approach to lattice field theory. Accurate Monte Carlo simulations can be performed and compared to analytical predictions, e.g., strong coupling computations. Fermions can be implemented directly and need not be integrated out, thus avoiding a nonlocal determinant; for certain models in $1+1$ dimensions, like the one studied here, they present no additional costs compared to bosons. It should be observed however, that in higher dimension fermions lead to sign problems which need to be solved [8]. Another important advantage of the Hamiltonian formalism is the possibility of preserving exactly a 1-dimensional supersymmetry algebra.

In the light of the encouraging preliminary results presented here, we plan to extend our study in several directions. We are computing the strong-coupling expansion, and we will study the weak-coupling expansion and the perturbative continuum limit. We will investigate numerically the transition from strong to weak coupling, looking at the pattern of supersymmetry breaking.

\section{REFERENCES}

1. J. Kogut, L. I. Susskind, Phys. Rev. D11 (1975) 395; J. Kogut, Rev. Mod. Phys. 51 (1979) 659.

2. W. von der Linden, Phys. Rept. 220 (1992) 53.

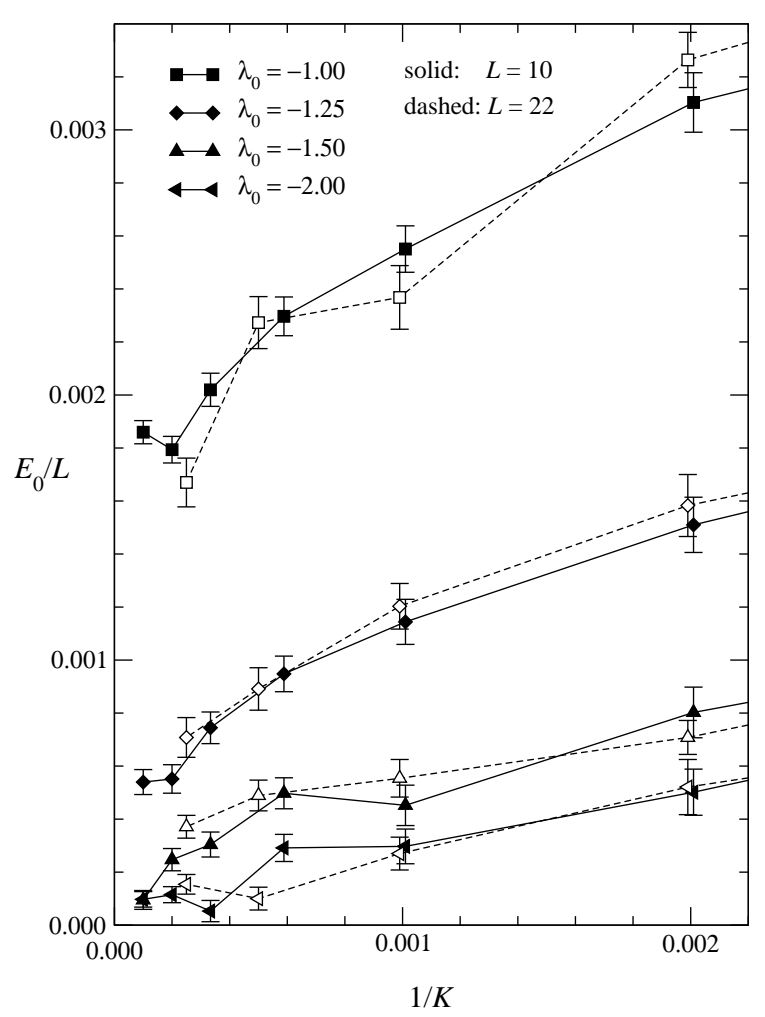

Figure 2. $E_{0} / L$ vs. $1 / K$ for $V=\phi^{2}+\lambda_{0}$.

3. S. Elitzur, E. Rabinovici, A. Schwimmer, Phys. Lett. B119 (1982) 165.

4. J. Ranft, A. Schiller, Phys. Lett. B138 (1984) 166.

5. M. Beccaria, M. Campostrini, A. Feo, heplat/0109005, to be published in Proc. of the Quantum Monte Carlo meeting, Trento, Italy, July 3-6, 2001.

6. E. Witten, Nucl. Phys. B188 (1981) 513; Nucl. Phys. B202 (1982) 253.

7. M. Beccaria, Phys. Rev. D61 (2000) 114503.

8. E. Y. Loh et al., Phys. Rev. B41 (1990) 9301; S. Chandrasekharan, U.-J. Wiese, Phys. Rev. Lett. 83 (1999) 3116. 